$\angle B L--38631$

LBL-38631

UL. 404

CONF- $960748-1$

ERNEST ORLANDO LAWRENCE

BERKELEY NATIONAL LABORATORY

Pressure Dependence of Se

Absorption Lines in AlSb

L. Hsu, E.E. Heller, and A.K. Ramdas

Materials Sciences Division

Center for Advanced Materials

September 1996

Presented at the

Seventh International

Conference on

Shallow-Level Centers

in Semiconductors

Amsterdam, The Netherlands,

July 17-19, 1996,

and to be published in

the Proceedings

DISTRIBUTION OF THIS DOCUMEN is UNMUTED of

MASTER 


\section{DISCLAIMER}

This document was prepared as an account of work sponsored by the United States Government. While this document is believed to contain correct information, neither the United States Government nor any agency thereof, nor The Regents of the University of California, nor any of their employees, makes any warranty, express or implied, or assumes any legal responsibility for the accuracy, completeness, or usefulness of any information, apparatus, product, or process disclosed, or represents that its use would not infringe privately owned rights. Reference herein to any specific commercial product, process, or service by its trade name, trademark, manufacturer, or otherwise, does not necessarily constitute or imply its endorsement, recommendation, or favoring by the United States Government or any agency thereof, or The Regents of the University of California. The views and opinions of authors expressed herein do not necessarily state or reflect those of the United States Government or any agency thereof, or The Regents of the University of California.

Ernest Orlando Lawrence Berkeley National Laboratory

is an equal opportunity employer. 


\section{DISCLAIMER}

Portions of this document may be illegible in electronic image products. Images are produced from the best available original document. 
LBL-38631

UC-404

\title{
Pressure Dependence of Se Absorption Lines in AlSb
}

\author{
L. Hsu ${ }^{1,3}$ E.E. Haller, 2,3 and A.K. Ramdas ${ }^{4}$ \\ ${ }^{1}$ Department of Physics \\ University of California \\ Berkeley, California 94720 \\ 2Department of Materials Science and Mineral Engineering \\ University of California \\ Berkeley, California 94720 \\ 3Materials Sciences Division \\ Ernest Orlando Lawrence Berkeley National Laboratory \\ University of California \\ Berkeley, California 94720 \\ ${ }^{4}$ Department of Physics \\ Purdue University \\ Lafayette, Indiana 47907
}

September 1996

This work was supported in part by the Director, Office of Energy Research, Office of Basic Energy Sciences, Materials Sciences Division, of the U.S. Department of Energy under Contract No. DE-AC03-76SF00098, and by the National Science Foundation Grant No. DMR-94 17763. 


\title{
PRESSURE DEPENDENCE OF Se ABSORPTION LINES IN AISb
}

\author{
L. HSU'3 and E. E. HALLER 23 \\ 'Dept. of Physics, University of California, Berkeley, CA 94720 \\ ${ }^{2}$ Dept. of Materials Science and Mineral Engineering, University of California, Berkeley, \\ CA 94720 \\ 'Materials Science Division, Lawrence Berkeley National Laboratory, Berkeley, CA 94720
}

A. K. RAMDAS

Dept. of Physics, Purdue University, Lafayette, IN 47907

\begin{abstract}
Using far infrared absorption spectroscopy, we have investigated electronic transition spectra of Se donors in AISb as a function of hydrostatic pressure. At least two distinct ground to bound excited state transition lines, which depend quadratically on the pressure, can be seen. At pressures between 30 and $50 \mathrm{kbar}$, evidence of an anti-crossing between one of the electronic transitions and a peak which we attribute to the 2 zone center LO phonon mode can be seen.
\end{abstract}

\section{Introduction}

Despite its similarity to GaAs and GaP, two technologically important materials, relatively little is known regarding AISb, especially with regard to its dopants. The study of impurities in AlSb, an indirect gap III-V semiconductor, has been hindered by two major factors. First of all, the quality and purity of available AlSb material is in general rather poor. Most AlSb is grown by the Czochralski method, where carbon contamination from the crucible has so far prevented very pure crystals from being produced. Secondly, AlSb is extremely hygroscopic. Crystals of AlSb left out in the open disintegrate into powder in a matter of weeks. Thus, surface preparation of this material for optical experiments is not as straightforward as with other materials. Lastly, it has been suggested that the conduction band minimum of $\mathrm{AlSb}$, found in the $\langle 100\rangle$ direction, has serious deviations from the model of the simple parabolic minimum which has worked so well in the cases of $\mathrm{Si}$ and $\mathrm{Ge}^{12}$. Arising from the lack of inversion symmetry within the crystal, the band minimum instead has a "camel's back" structure, for which one is unable to define a particular longitudinal effective mass for conduction electrons. As was the case of GaP, evidence for this deviation has been found in the absorption spectra of donors in AlSb, in this case Se and Te, which could not be fit to Faulkner's model for donors in the elemental semiconductors ${ }^{3}$. Using a simple variational calculation and a nonparabolic dispersion relation however, it has been claimed that the spacings of the donor absorption lines can be predicted quite well'. No detailed analysis of the donor states has yet been published. 


\section{Experimental Techniques}

The use of hydrostatic pressure as a variable in the study of semiconductors has been a well-known technique for over ten years'. Now, we have applied this technique to the study of Se donors in AlSb. The samples used in our experiment were small pieces of Se-doped AISb, cut from single-crystal ingots grown at Bell and Howell in the 1960's. At room temperature, the net carrier concentration of the samples was about $10^{16} \mathrm{~cm}^{-3}$. After lapping the material to a thickness of $50 \mu \mathrm{m}$ and using methanol and very fine grit to polish the surfaces to a mirror-like finish, an ultrasonic grinder was used to cut out disks about $300 \mu \mathrm{m}$ in diameter. The samples were then loaded into a modified Merrill-Basset diamond anvil cell (DAC) ${ }^{6}$ along with a few grains of ruby. Liquid nitrogen, which has been shown to give hydrostatic environments up to $130 \mathrm{kbar}$ was used as a pressure medium? ${ }^{7}$. The wavelength shift of one of the ruby fluorescence lines was used to measure the pressure in the cell at room temperature for purposes of changing the pressure. To determine the pressure in the cell at low temperatures, however, the frequency of a vibrational mode of residual $\mathrm{CO}_{2}$ dissolved in the liquid nitrogen was used.

Measurements of the absorption spectra were performed at liquid helium temperatures using a $\mathrm{Ge}: \mathrm{Cu}$ photoconductor. Located inside an integrating optical cavity, the detector was mounted directly behind the cell and a light concentration cone was placed in front of the DAC to focus as much light as possible though the diamonds and onto the detector. The entire setup was placed inside a Janis Super VP cryostat and infrared transmission measurements were performed using a Digilab FTS-80 Fourier-Transform spectrometer ${ }^{6}$. Using a $\mathrm{KBr}$ beamsplitter, measurements could be performed in the energy range from $450 \mathrm{~cm}^{-1}$ up to $4000 \mathrm{~cm}^{-1}$.

Since the signals we expect from impurity spectroscopy through a DAC are small, a good reference spectrum was needed to produce an absorption spectrum which was as free from instrumental and bulk material features as possible. The existence of DX centers in AlSb made this possible. Although DX centers were first discovered in GaAs and have been more extensively sudied in that material ${ }^{9,10}$, they are also found in AlSb, where at atmospheric pressure the DX center is the most stable form of Se and $\mathrm{Te}$ impurities". One of the most striking characteristics of DX centers, and the property which we exploited, is the persistent photoionization they show at low temperatures. If the DX centers in a crystal are exposed to photons of sufficient energy, they can be transformed back into the normal shallow donor configuration. At low temperatures $(<-100 \mathrm{~K})$, a thermal barrier prevents their return to the DX state.

Since DX centers are deep states which are spectroscopically inactive in the energy range with which we are concerned, we were able to use each sample as its own reference. For each absorption spectrum shown, the sample was cooled under 
pressure in the dark, allowing the impurities to assume their DX form, and a reference spectrum was taken. The light incident on the sample during this process did not possess enough energy to perturb the DX centers. Light of much highèr energy was then shined on the sample using an AlGaAs diode, converting the impurities from their DX form into their shallow donor configuration, and a second spectrum was taken. Taking the logarithm of the ratio of the post- and pre-light spectra produced the absorption curves.

\section{Results}

In Figure 1A, we see the absorption spectrum of Se donors in AlSb at atmospheric pressure. Two prominent peaks can be seen. First observed in 1968, the lower energy line was attributed to a $1 s\left(A_{1}\right)$ to $1 s\left(T_{1}\right)$ transition and the higher energy line to a $1 s\left(A_{1}\right)$ to $2 \mathrm{p}_{ \pm}$transition ${ }^{3}$.

Comparing this spectrum to those of donors in GaAs at pressures above which it is an indirect gap semiconductor (in the future when we refer to GaAs, we will always mean at pressures above which it has an indirect gap) and in GaP, we see some similarities, the most prominent of which is that only a few electronic transitions are $\operatorname{seen}^{12.13}$. This can be attributed to the camel's back structure of the conduction band

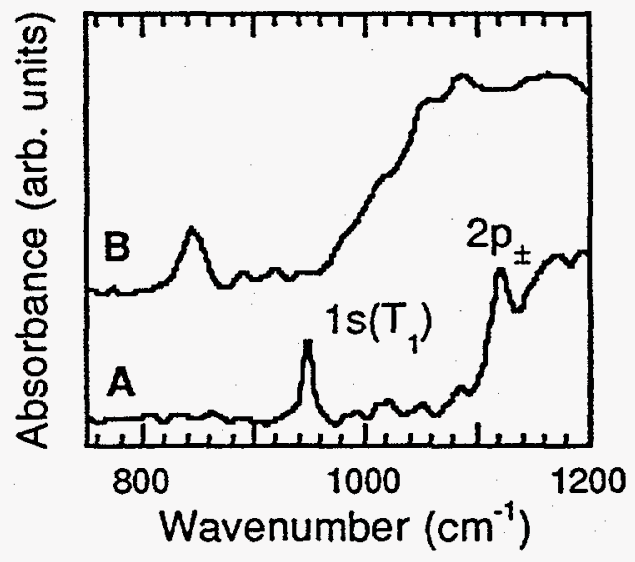

Figure 1-Absorption spectrum of AlSb:Se at atmospheric pressure (A) and at $7 \mathrm{kbar}(B)$. showing disappearance of $2 p_{ \pm}$line.

minimum. In contrast to the case of the parabolic band minimum where there are an infinite number of bound excited states, in the camel's back case it has been shown that there may be as few as one bound excited state of the donor electron. The higher excited states are auto-ionized and lie within the conduction band, where they may or may not be seen in the continuum. In the spectrum shown for AlSb, the positions of the two transitions seen indicate that these bound excited states are already very close to the ionization energy of the Se donor, as shown by the position of the continuum absorption.

Upon the application of pressure, these absorptions decrease in energy at different rates. As can be seen in Figure 1B, at 7 kbar pressure, the continuum has moved faster than the higher energy transition line, which has almost completely 


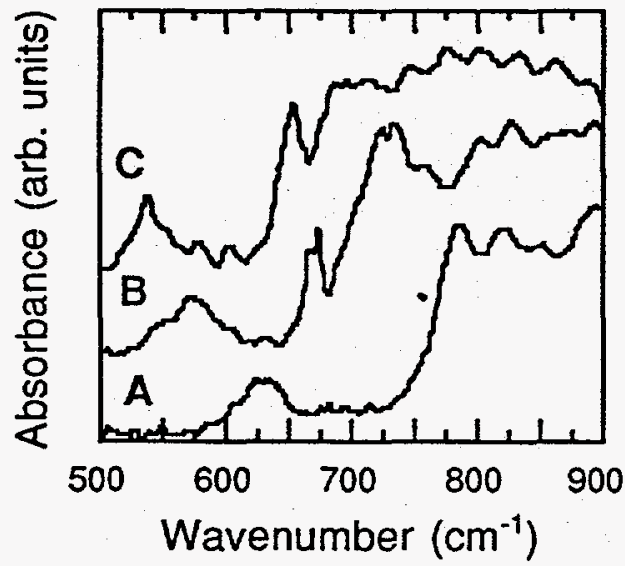

Figure 2-Absorption spectra of AlSb:Se at 29 kbar (A), 43 kbar (B), and 50 kbar (C), showing emergence of two phonon line. disappeared. At still higher pressures, however, it re-emerges. Such behavior can easily be explained by the warping of the conduction band minimum. It is a well known fact that the energies of the conduction band minima shift with respect to the top of the valence band as a function of hydrostatic pressure. However, here we see that the shape of the minimum warps as well, a change in the effective masses leading to a change in the ionization energies of the bound excited states relative to the band edge.

At still higher pressures, we see

the emergence of a completely new peak which was absent at lower pressures (Figure 2). This new peak can be seen faintly at $29 \mathrm{kbar}$. It then grows in size with higher pressures until it is as large as the original $1 \mathrm{~s}$ to $2 \mathrm{p}_{ \pm}$peak and becomes only a single peak. Finally, around $59 \mathrm{kbar}$, the continuum absorption catches up to and completely swallows this new peak.

If we plot the peak positions as a function of pressure, we produce the graph shown in Figure 3. As is evident from the plot, the transition lines have a quadratic dependence on the pressure. The two donor absorption lines do not have the same pressure dependence, the energy of the $2 p_{ \pm}$ line decreasing faster than that of the $1 s\left(T_{1}\right)$ line. The most interesting behavior, however, occurs between 30 and $50 \mathrm{kbar}$ and involves the $1 \mathrm{~s}$ to $2 \mathrm{p}_{ \pm}$ transition and the peak which starts to appears at $30 \mathrm{kbar}$. As the pressure increases, the two peaks approach each other until they are about 40 wavenumbers apart at roughly 40 kbar. Unfortunately, the $1 \mathrm{~s}$ to $2 \mathrm{p}_{ \pm}$

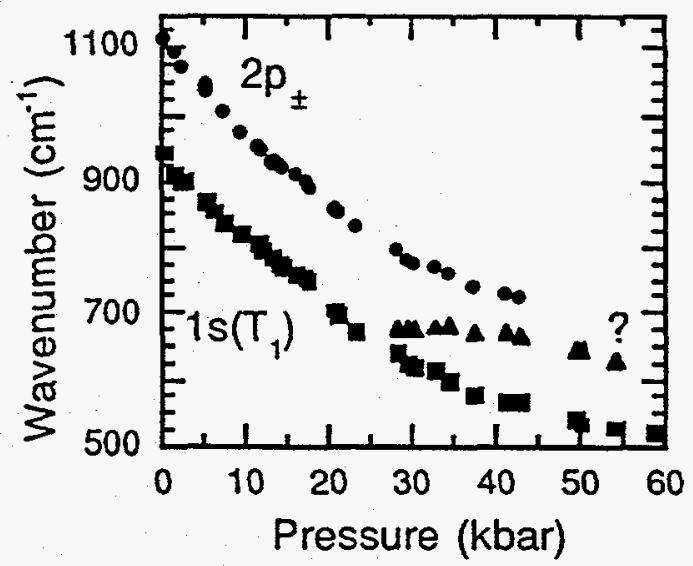

Figure 3--Pressure dependence of the major peaks in the AISb:Se absorption spectrum. 
absorption line begins to broaden severely at $30 \mathrm{kbar}$, becoming lost in the continuum above $40 \mathrm{kbar}$.

\section{Interpretation}

One possible model which could explain this behavior involves an anti-crossing between the two absorptions. In Figure 4, we plot the experimental data of interest along with the energy of the $2 \mathrm{LO}$ zone center phonon mode and what we believe to be the energy of the unperturbed is to $2 p_{ \pm}$electronic transition as a function of pressure. Based on the positions of these two lines, we see that the explanation of an anti-crossing of these two absorptions is a plausible one. The position of the two zone center LO phonon mode seems a bit high in the figure. However, the exact energy and pressure dependence of this two phonon lattice mode is unknown and was estimated from the data for a single LO zone center phonon $^{14}$. The peak which mysteriously appears is phonon-like below $40 \mathrm{kbar}$ and thus starts out small, as this absorption is present in both the reference and sample spectra. As the pressure is increased, it gains strength as it becomes more and more electronic transition-like. Because the electronic transition is not present in the reference sample, we are now able to see this

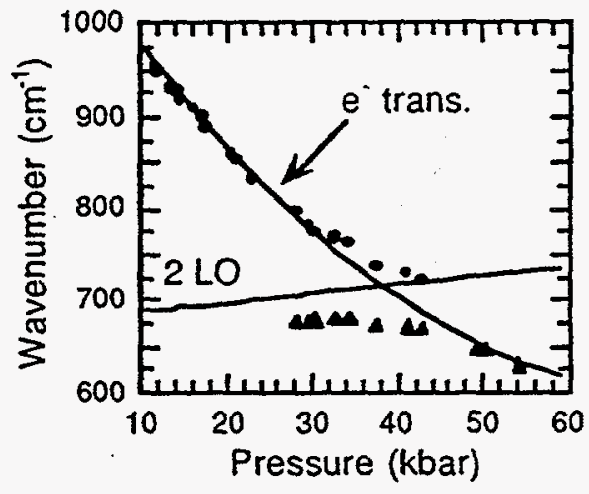

Figure 4-Plot of anti-crossing region beween electronic transition and two-phonon absorption. The lines represent unperturbed two-phonon and electronic tansition energies. absorption. In contrast, the upper branch, which is originally an electronic transition, starts out strong, but is then considerably broadened by its interaction with the 2 phonon mode and becomes more phonon-like. Above $40 \mathrm{kbar}$, it becomes extremely difficult to see this line due to its broadness and the large absorption due to the continuum. The interaction between the two absorptions is most likely due to the macroscopic electric field of the LO phonons. Again, we stress that this is only one possible explanation. More work will be required to either verify or disprove this model.

In conclusion, we have studied the absorption spectrum of Se impurities in AlSb as a function of pressure, identifying two peaks in the spectra as due to transitions from the ground state to $1 s\left(T_{1}\right)$ and $2 p_{ \pm}$bound excited states. In addition to finding the spectrum to be similar to that of other donors in III-V indirect gap semiconductors, we also see that the band minimum warps as a function of pressure, 
changing the effective mass and thus the line spacings. To explain the appearance of a third peak at $30 \mathrm{kbar}$, we propose a model in which there is an anti-crossing of the two-phonon absorption and the $1 \mathrm{~s}$ to $2 \mathrm{p}_{ \pm}$electronic transition. The phonon absorption gradually increases in strength, becoming more electronic transition-like while the electronic transition line becomes more phonon-like, broadening out before disappearing into the continuum. The reason why the two phonon absorption interacts with the $2 \mathrm{p}_{ \pm}$transition, but not the $1 s\left(T_{1}\right)$ transition line will require further study.

\section{Acknowledgments}

This work was supported in part by NSF grant DMR-94 17763 and in part by the Director, Office of Energy Research, Office of Basic Energy Sciences, Materials Science Division of the U.S. Dept. of Energy under Contract No. DE-AC0376 SF00098.

\section{References}

1. A. A. Kopylov, Solid State Comm. 56, 1 (1985).

2. R.A. Faulkner. Phys. Rev. 184, 713 (1969).

3. B. T. Ahlburn, A. K. Ramdas, Phys. Rev. 167, 717 (1968).

4. G. F. Glinski, A. A. Kopylov, A. N. Pikhtin, Solid State Comm. 30, 631 (1979).

5. C. J. Armistead, F. Kuchar, S. P. Nadja, S. Porowski, C. Sotomayor-Torres, R. A. Stradling, Z. Wasilewski, Proc. 17th ICPS, 1047 (1984).

6. J. A. Wolk, M. B. Kruger, J. N. Heyman, W. Walukiewicz, R. Jeanloz, E. E. Haller, Phys. Rev. Lett. 66, 774 (1991).

7. R. LeSar, S. A. Ekberg, L. H. Jones, R. L. Mills, L. A. Schwalbe, D. Schiferl, Solid State Comm. 32, 131 (1979).

8. M. D. McCluskey, L. Hsu, L. Wang, E. E. Haller, submitted to Phys. Rev. B.

9. D. J. Chadi, K. J. Chang, Phys. Rev. B 39, 10063 (1989).

10. J. Zeman, M. Zigone, G. Martinez, Phys. Rev. B 51, 17551 (1995).

11. P. Becla, A. Witt, J. Lagowski, W. Walukiewicz, Appl. Phys. Lett. 67, 395 (1995).

12. A. A. Kopylov, A. N. Pikhtin, Solid State Comm. 26, 735 (1978).

13. L. Hsu, E. E. Haller, S. Zehender, E. Bauser, submitted to Phys. Rev. Lett. 14. S. Ves, K. Strössner, M. Cardona, Solid State Comm. 57, 483 (1986). 